\title{
Systèmes agroforestiers à cacaoyers et transition capitaliste : l'exemple du Centre-Cameroun
}

Philippe Pédelahore

Cirad

UMR Innovation

TAC-85/15

73, rue Jean-François Breton 34398 Montpellier Cedex 5 France

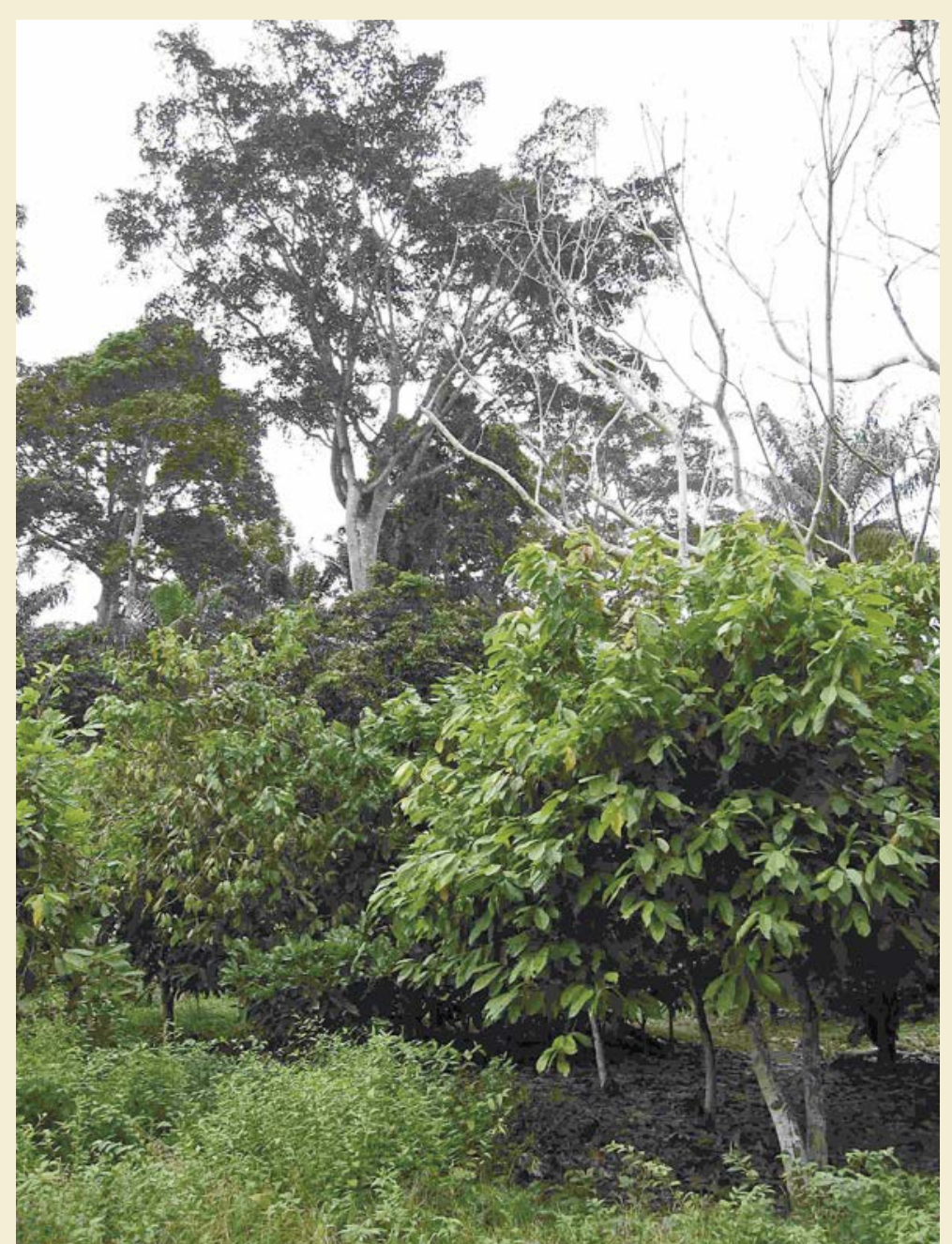

Photo 1.

Agroforêt à base de cacao de la zone d'Obala. Photo P. Pédelahore. 


\section{RÉSUMÉ}

\section{SYSTÈMES AGROFORESTIERS \\ À CACAOYERS ET TRANSITION \\ CAPITALISTE : L'EXEMPLE \\ DU CENTRE-CAMEROUN}

La production cacaoyère camerounaise est issue de systèmes agroforestiers complexes. Après une longue période de stagnation entre 1963 et 1993, cette production a doublé entre 1993 et 2013. Quels sont les exploitants agricoles qui portent ces dynamiques d'accroissement de la production cacaoyère? Sur quels moyens de production et stratégies se sont-ils appuyés pour parvenir à relancer cette filière et à en doubler la production sur une aussi courte période ? Pour contribuer à apporter des éléments de réponse à ces questions, des entretiens semi-directifs ont été conduits auprès de 82 planteurs représentatifs de la diversité du principal bassin de production, dans la région Centre. Les résultats obtenus montrent que l'essor de la production cacaoyère de la zone étudiée repose en grande partie sur la montée en puissance des formes patronale et capitaliste de la production agricole. Ces planteurs d'un genre nouveau investissent d'importants volumes de capitaux financiers d'origine urbaine dans la création de grandes plantations dans les zones de fronts pionniers. Ces résultats incitent à analyser l'évolution de ces systèmes agroforestiers en portant une plus grande attention qu'auparavant aux interactions entre les différentes formes de production (familiale, patronale, capitaliste) et entre les trajectoires d'accumulation en capital qui se développent en milieu rural et en milieu urbain.

Mots-clés : agroforesterie, cacao, accumulation en capital, Cameroun.

\section{ABSTRACT}

\section{COCOA-BASED AGROFORESTRY AND THE TRANSITION TO A CAPITALIST ECONOMY IN CENTRAL CAMEROON}

Cocoa production in Cameroon has developed from complex agroforestry systems. After a long period of stagnation from 1963 to 1993, production doubled over the next two decades. Which are the farmers driving the growth and dynamics of cocoa production? What are the strategies and production methods behind their success in reviving the sector and doubling production over such a short time? To find answers to these questions, we organised semi-directive interviews with 82 farmers representing the different types of plantations in central Cameroon, the country's main cocoa-growing region. Our results show that the production boom in the study area is largely based on the rise of capitalistic farming based on salaried labour, with a new type of plantation owner investing large amounts of financial capital, of urban provenance, to create large-scale plantations along pioneer fronts. These findings led us to analyse developments in these agroforestry systems with a closer focus on interactions between the different forms of production (family farms, salaried labour, capitalist ventures) and between the new patterns of capital accumulation developing in both rural and urban areas.

Keywords: agroforestry, cocoa, accumulation of capital, Cameroon

\section{RESUMEN}

\section{SISTEMAS AGROFORESTALES CACAOTEROS Y TRANSICIÓN CAPITALISTA: EJEMPLO DEL CENTRO DE CAMERÚN}

La producción cacaotera camerunesa procede de sistemas agroforestales complejos. Tras un largo período de estancamiento entre 1963 y 1993, dicha producción se duplicó entre 1993 y 2013. ¿Qué tipo de productores generan estas dinámicas de incremento de la producción cacaotera? ¿En qué medios de producción y estrategias se han basado para lograr reactivar el sector y duplicar la producción en tan poco tiempo? Para intentar aportar elementos de respuesta a estos interrogantes, se realizaron entrevistas semidirectivas a 82 cultivadores representativos de la diversidad de la principal área de producción en la región del Centro. Los resultados obtenidos muestran que el auge de la producción cacaotera de la zona estudiada se basa en gran parte en la expansión de formas patronales y capitalistas de producción agrícola. Estos nuevos cultivadores invierten importantes volúmenes de capitales financieros de origen urbano en la creación de grandes plantaciones en zonas de frentes pioneros. Estos resultados inducen a analizar la evolución de estos sistemas agroforestales prestando mayor atención que antes a las interacciones entre las distintas formas de producción (familiar, patronal, capitalista) y entre las trayectorias de acumulación de capital que se desarrollan en ámbitos rurales y urbanos.

Palabras clave: agroforestería, cacao, acumulación de capital, Camerún. 


\section{Les systèmes agroforestiers à cacao au Cameroun : un cas intéressant à étudier}

L'agroforesterie est souvent présentée comme l'une des voies possibles pour l'intensification écologique des systèmes de cultures, qui consiste à augmenter les rendements de manière naturelle en utilisant au maximum les fonctionnalités écologiques et biologiques des écosystèmes (Griffon, 2002). Elle permettrait l'amélioration des revenus et de la résilience des exploitants agricoles familiaux face aux aléas économiques et climatiques (Garrity, 2004). Pour être en mesure d'accompagner ou d'orienter le développement de l'agroforesterie, il est utile de comprendre les dynamiques historiques et actuelles de récession, de maintien ou d'extension de ces systèmes de culture (Eloy, 2008) et donc d'expliciter les stratégies des exploitants agricoles qui délaissent, maintiennent ou développent les différents types de systèmes agroforestiers (SAF) (De Souza et al., 2012).

Les systèmes agroforestiers à base de cultures pérennes comme le caféier ou le cacaoyer représentent une part importante des surfaces agroforestières mondiales et concernent un nombre considérable d'exploitants familiaux. En Afrique, le Cameroun a historiquement développé sa production cacaoyère dans des systèmes agroforestiers complexes (Sonwa et al., 2003) où le cacao est généralement placé sous l'ombrage d'espèces forestières et est complanté avec une grande diversité de plantes utiles (agrumes, mangue, safou, bananier plantain...). Ce qui rend intéressant l'analyse de l'évolution de la situation camerounaise, c'est que les SAF à base de cacao, après avoir présenté une longue période de stagnation entre 1963 et 1993, ont connu entre 1993 et 2011 un essor spectaculaire. Bien qu'il n'y ait pas de données statistiques postérieures à 1984 sur l'évolution des surfaces des SAF à base de cacao, il est possible de s'appuyer sur l'analyse de l'évolution de la production cacaoyère issue de ces SAF pour rendre compte de l'importance de ces bouleversements. Cette production, qui était de l'ordre de 110000 tonnes durant la période 1963-1993 (Varlet, 1997), a dépassé les 200000 tonnes lors des campagnes agricoles de 2009, 2010 et 2011 (CTA, 2012).

Quels sont les exploitants agricoles qui portent ces dynamiques d'accroissement de la production cacaoyère ? Sur quels moyens de production et sur quelles stratégies se sont-ils appuyés pour parvenir à relancer cette filière et à en doubler la production sur une aussi courte période ?

Des enquêtes menées en 2010 dans le principal bassin de production de cacao apportent des éléments de réponse. Ceux-ci sont discutés en regard des données statistiques et bibliographiques disponibles, et en les replaçant dans le cadre plus général de l'analyse des dynamiques des SAF à base de plantes pérennes et des différentes formes de production agricole.

\section{Une étude basée sur la caractérisation de deux sites représentatifs du bassin cacaoyer du Centre-Cameroun}

Deux zones représentatives de la diversité des dynamiques cacaoyères du Centre-Cameroun, principal bassin cacaoyer du pays, ont été retenues pour les besoins de l'enquête.

La première s'étend sur l'arrondissement d'Obala (figure 1), situé dans le département de La Lékié. Cet arrondissement de $470 \mathrm{~km}^{2}$ est situé à $40 \mathrm{~km}$ au Nord de Yaoundé. Il est relié à la capitale par une route goudronnée en bon état. C'est une zone cacaoyère ancienne et densément peuplée (> 100 habitants $/ \mathrm{km}^{2}$ ) (PNUD, 2000). Les habitants relèvent quasi exclusivement de l'ethnie autochtone Eton, et tous les planteurs interrogés appartiennent à cette ethnie. Les cacaoyères existantes dans cette zone ont généralement plus de 50 ans, même si les pratiques techniques conduisent à un remplacement progressif et constant des pieds les plus âgés (Jagoret, 2011). Les systèmes agroforestiers de cette zone sont des systèmes complexes et multistrates qui représentent bien l'archétype de la cacaoculture camerounaise décrite en introduction.

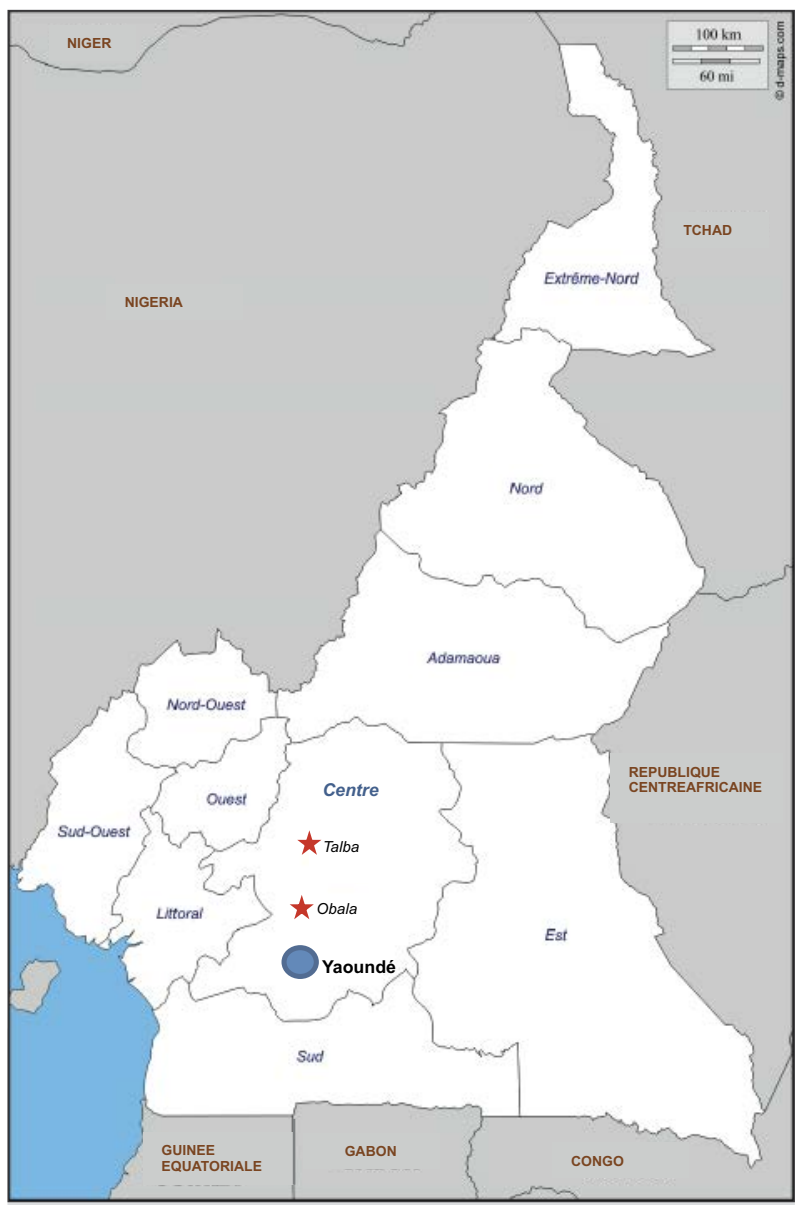

Figure 1.

Localisation des zones d'Obala et de Talba.

Source du fond de carte : http://agcc.fr 


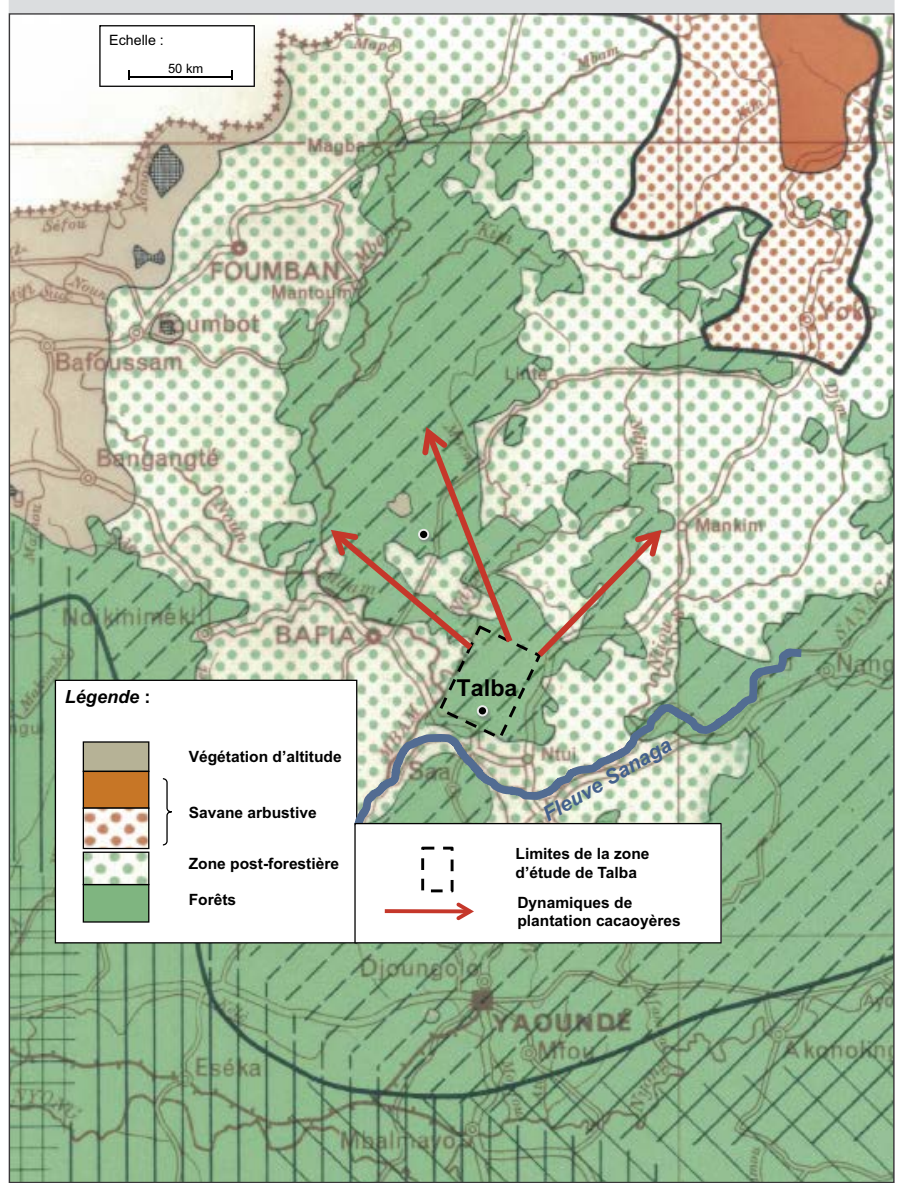

\section{Figure 2.}

Localisation de la zone de Talba et des dynamiques de plantation cacaoyères.

Source du fond de carte (Orstom, 1959).

La forme patronale se caractérise par une main-d'œuvre salariée supérieure à la main-d'œuvre familiale et par le fait que le propriétaire de la plantation réside sur son exploitation. La forme capitaliste est celle où la main-d'œuvre salariée est supérieure à la main-d'œuvre familiale et où le propriétaire de la plantation ne réside pas sur son exploitation, ce qui le conduit à en confier la gestion à un chef d'exploitation salarié.

\section{Résultats}

\section{Talba (14 autochtones +32 allochtones).}

La seconde phase a reposé sur des entretiens semidirectifs (Olivier de Sardan, 1995) auprès de ces 82 planteurs. Ils ont précisé leurs stratégies et trajectoires d'accumulation (Gafsi, 2006) en surfaces cacaoyères, l'origine de leurs ressources foncières et financières, les types de maind'œuvre utilisés sur leur exploitation, et les rendements obtenus au cours des trois dernières campagnes agricoles. Sur les 82 planteurs enquêtés, seuls 56 ont souhaité ou pu renseigner cette dernière question.

Les traitements statistiques des données quantitatives présentés ont utilisé successivement l'analyse de variance (Anova) et le test de Fischer. La classification des formes de production agricole utilisée dans cette étude a repris celle proposée par Bergeret et Dufumier (2002). Dans la forme familiale, la main-d'œuvre familiale utilisée sur l'exploitation est supérieure (en nombre d'heures travaillées/an) à la maind'œuvre salariée, que celle-ci soit permanente ou temporaire, et le propriétaire de la plantation réside sur son exploitation.
Les systèmes agroforestiers (tableau I) de la zone d'Obala se caractérisent par la prédominance des petits et moyens planteurs, tant du point de vue numérique (97\%) que de celui de la part relative des surfaces cacaoyères qu'ils contrôlent ( 86 \%). La surface moyenne est de 2,44 ha de cacao par exploitation. Les systèmes agroforestiers de la zone de front pionnier de Talba se caractérisent au contraire par la prédominance des grands et très grands planteurs. Même s'ils ne représentent que $30 \%$ des planteurs de la zone de Talba, ils contrôlent 75 \% des surfaces cacaoyères. La surface moyenne est de 8,21 ha par exploitation. Les grands et les très grands planteurs de la zone de Talba sont majoritairement (93\%) des allochtones dont $93 \%$ sont originaires du département de La Lékié où est située la zone

\section{Un développement des exploitations de grande taille dans les fronts pionniers}


d'Obala. Ces allochtones appartiennent aux ethnies Eton et Manguissa. Ces deux ethnies ont des liens généalogiques anciens avec les autochtones de la zone de Talba, qui sont de l'ethnie Sanaga, et qui détiennent les droits fonciers coutumiers sur les terres de cette zone de front pionnier.

Les rendements moyens en cacao marchand obtenus par l'ensemble des planteurs de la zone d'Obala (248 kg/ha) sont statistiquement inférieurs à ceux obtenus par les planteurs de la zone de Talba $(524 \mathrm{~kg} / \mathrm{ha})$.

\section{Une marchandisation de la terre et de la force de travail}

Le tableau II présente les modalités d'accès à la ressource foncière pour les planteurs de chacune des deux zones. Il sépare pour la zone de Talba l'analyse des réponses apportées par les planteurs autochtones de celles des planteurs allochtones. Le total des réponses dépasse 100 \% puisqu'un même planteur peut avoir obtenu de la terre par différentes modalités.

Les petits et moyens exploitants de la zone d'Obala ont majoritairement (97\%) eu accès à la terre par héritage. Le droit de la hache, en vigueur dans cet arrondissement jusqu'au début des années 1960, a disparu en même temps que croissaient la densité de population et l'occupation du territoire. Les $17 \%$ ayant déclaré avoir acheté des terres ne doivent pas faire penser qu'il existe un important marché foncier dans cet arrondissement. En effet, la majorité des terres achetées concernent des zones de fronts pionniers situées hors de l'arrondissement d'Obala, au Nord (zone de Talba et de Ntui) et à l'Est (zone de Mbandjok) de celui-là. Malgré un prix de vente élevé (de plusieurs centaines de milliers de FCFA/ha à plusieurs millions de FCFA/ha), il apparaît dans cet arrondissement une patrimonialisation de la terre qui soustrait cette ressource à l'influence des règles du marché. La majorité des chefs de famille refusent l'idée même de vendre leurs terres et encore plus leurs parcelles de cacao. Certaines chefferies de cet arrondissement, comme celle de Minkama, ont même décidé d'interdire les ventes de terre.

Pour la zone de Talba, c'est bien l'achat qui est la modalité dominante d'accès à la terre puisque les allochtones représentent $85 \%$ du nombre total des planteurs de cette zone. Certains des premiers migrants installés dans des lieux éloignés, isolés et inhabités ont pu, lors de l'ouverture de ce front pionnier, s'approprier des terres par le droit de la hache (22\%). Cette modalité a ensuite rapidement et pratiquement disparu. Les quelques allochtones (9\%) ayant déclaré avoir reçu de la terre par héritage sont trois jeunes planteurs que leur vieux père, encore en vie, a récemment installés sur une partie des terres par lui achetées. Ces achats se font auprès des « chefs de terre » autochtones. Le montant moyen est de

Tableau I.

Importance relative des différents types de planteurs en fonction de leur nombre et des surfaces des cacaoyères qu'ils contrôlent dans la zone.

\begin{tabular}{|c|c|c|c|c|}
\hline Surface de cacaoyère possédée (ha) & $\begin{array}{c}\text { Petit planteur } \\
0,1<x \geq 2\end{array}$ & $\begin{array}{c}\text { Planteur moyen } \\
2<x \geq 6\end{array}$ & $\begin{array}{c}\text { Grand planteur } \\
6<x \geq 30\end{array}$ & $\begin{array}{c}\text { Très grand planteur } \\
\qquad x>30\end{array}$ \\
\hline \multicolumn{5}{|l|}{ Zone d'Obala } \\
\hline$\%$ nombre de planteurs & 59 & 38 & 2,9 & 0,1 \\
\hline Surface moyenne (ha/planteur) & 1 & 4 & 10 & 40 \\
\hline \% surface de cacaoyère & 24 & 62 & 12 & 2 \\
\hline \multicolumn{5}{|l|}{ Zone de Talba } \\
\hline$\%$ nombre de planteurs & 25 & 45 & 26 & 4 \\
\hline Surface moyenne (ha/planteur) & 1 & 4 & 16 & 50 \\
\hline \% surface de cacaoyère & 3 & 22 & 51 & 24 \\
\hline
\end{tabular}

Tableau II.

Modalités d'accès à la terre pour les planteurs d'Obala et de Talba.

\begin{tabular}{|c|c|c|c|c|}
\hline Zone & $\begin{array}{c}\text { Droit de la hache } \\
\text { (= droit du premier occupant) }\end{array}$ & Héritage & Achat & Total \\
\hline Obala (autochtones) & $0 \%$ & $97 \%$ & $17 \%$ & $124 \%$ * \\
\hline Talba (autochtones) & $0 \%$ & $100 \%$ & $14 \%$ & $114 \%$ \\
\hline Talba (allocthones) & $22 \%$ & $9 \%$ & $69 \%$ & $111 \%$ * \\
\hline
\end{tabular}


$50000 \mathrm{FCFA} /$ ha (80 euros/ha). Ce montant, en francs CFA courants, est le même depuis 1980, mais le marché foncier se déplace chaque année plus vers le nord de la zone de Talba au fur et à mesure que les migrants remplacent la forêt originelle par des systèmes agroforestiers à base de cacaoyers. Les entretiens réalisés avec les personnes-ressources et les parcours réalisés dans la zone de Talba indiquent que la grande majorité de cette zone a été occupée depuis les années 2000 par des SAF à cacaoyers. Le front pionnier aurait ainsi progressé d'une quarantaine de kilomètres vers le nord en 20 ans (1980-2000). Les entretiens réalisés indiquent également que cette dynamique d'achat de terres et de plantation concerne déjà un territoire beaucoup plus étendu que notre zone d'étude et que cette dynamique poursuit son développement vers le nord-ouest, le nord et le nord-est de notre zone d'étude (figure 2).

Le processus de marchandisation décrit pour la terre concerne également l'accès à la force de travail. Dans la zone d'Obala, la main-d'œuvre reste à $71 \%$ familiale ou basée sur l'entraide. La situation est inverse pour la zone de Talba où ce pourcentage concerne la main-d'œuvre extérieure à la famille (tableau III). Cette main-d'œuvre non familiale relève de divers statuts. Elle est majoritairement rémunérée à travers un paiement à la tâche (38\%), un salaire mensuel (9\%) ou un contrat de campagne (14\%) qui la lie pour toute une saison cacaoyère à un intermédiaire, qui joue le rôle de recruteur, et au planteur. Les surfaces cacaoyères en métayage ne représentent que 10 \% des surfaces cacaoyères totales de la zone. Cette main-d'œuvre non familiale est majoritairement anglophone. Elle est originaire des zones déshéritées et surpeuplées du département du Nord-Ouest (figure 1) et ses ressortissants sont appelés les "Bamenda » ou les « Mambila » par les planteurs.

La rémunération du travail à la tâche est pour la période 2010-2013 de l'ordre de 25000 FCFA/ha (40 euros/ha) pour le travail de désherbage des SAF à cacaoyers.

\section{Un essor des formes patronale et capitaliste de la production agricole}

Les 82 planteurs ont été classés en fonction de l'origine de leur force de travail (familiale ou salariée majoritaire) et leur lieu de résidence (sur l'exploitation ou en dehors de celle-ci). Cette classification renseigne sur l'importance relative des formes de production par zone (tableau IV). Elle montre que la marchandisation de la terre et de la force de travail agricole a conduit dans la zone de Talba à une montée en puissance des formes patronale et capitaliste de la production agricole. Elle montre également, lorsque l'on rapproche les chiffres et les données d'enquêtes résumées dans le tableau I et le tableau IV, qu'à partir d'une surface de SAF à cacaoyers de 5 à 6 ha, les exploitants utilisent de la main-d'œuvre majoritairement salariée et relèvent donc des formes patronale et capitaliste de la production agricole. Les petites et moyennes exploitations qui détiennent moins de 6 ha relèvent majoritairement des formes de production familiales.

\section{Une transition liée à l'investissement de capitaux financiers d'origine non agricole}

Les questions portant sur les statuts actuels et parcours professionnels des 82 exploitants enquêtés, et sur l'origine des capitaux financiers qu'ils ont investis dans la création et l'entretien de leurs plantations cacaoyères, montrent que la grande majorité des grands et des très grands planteurs tirent leurs capitaux financiers de revenus non agricoles. Ce sont leurs activités urbaines (propriétaires immobiliers, commerçants, responsables de compagnies de transport, hauts cadres affairistes du secteur public ou privé...) qui leur ont permis d'accumuler d'important volumes de capitaux financiers. C'est à partir de ces capitaux financiers que ces entrepreneurs agricoles d'un genre

Tableau III.

Importance relative des différents types de main-d'œuvre en pourcentage des surfaces cacaoyères pour les zones d'Obala et de Talba.

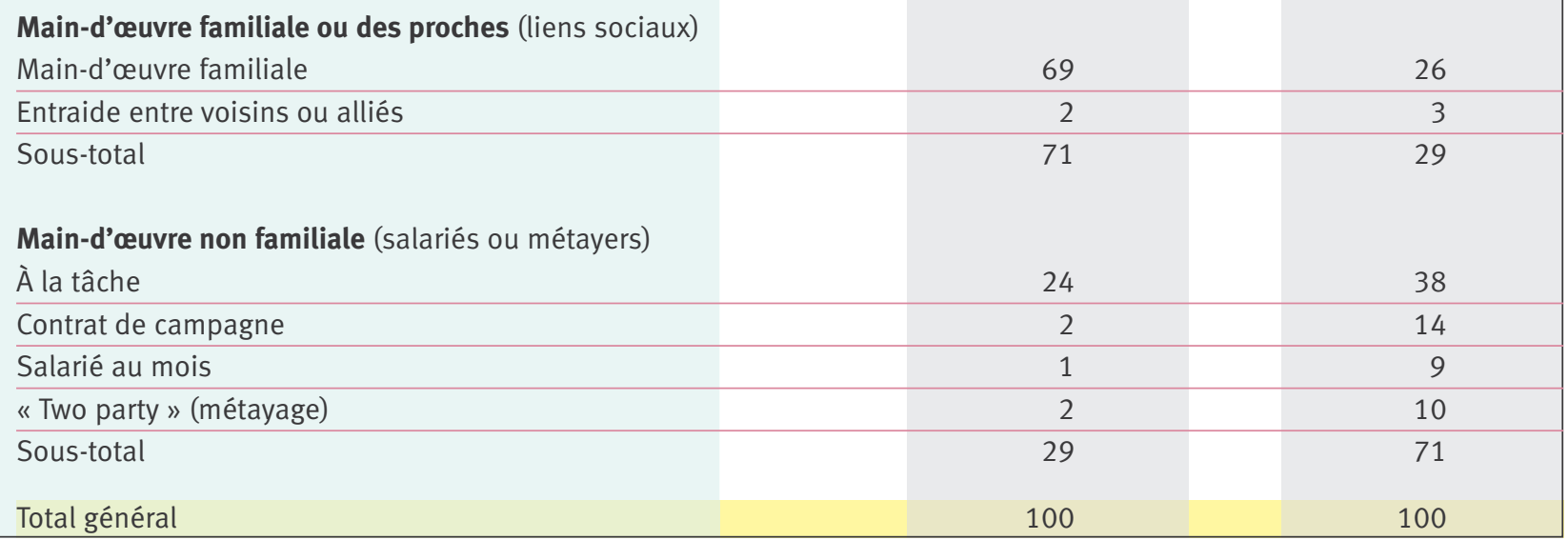


nouveau ont pu acquérir de la terre et rémunérer de la maind'œuvre agricole pour développer rapidement d'importantes surfaces cacaoyères. L'analyse des surfaces possédées en fonction des revenus des exploitants met en évidence l'importance du niveau des revenus non agricoles pour les capacités d'accumulation en surface de plantations cacaoyères chez les 82 exploitants interrogés (tableau V). Elle montre que les exploitants sans revenus non agricoles ou avec des revenus non agricoles faibles n'ont pu créer que des surfaces cacaoyères moyennes peu importantes s'élevant respectivement à 4,5 ha et 4,7 ha/exploitation. Par contre, les exploitants agricoles bénéficiant ou ayant bénéficié de revenus non agricoles moyens ou élevés ont pu atteindre des surfaces cacaoyères respectivement de l'ordre de 14,5 ha et 88 ha/exploitation, c'est-à-dire 3 à 20 fois plus importantes que les planteurs purs.

\section{Un contexte socio-économique favorable aux investisseurs urbains}

Les données recueillies en 2010 auprès des grands et des très grands planteurs de Talba sur les coûts d'installation et d'entretien d'une cacaoyère montrent que ceux-ci s'élèvent respectivement à 550000 FCFA/ha et à 250000 FCFA/ha/an. La main-d'œuvre représente $80 \%$ des coûts d'installation contre $9 \%$ seulement pour l'achat de la terre. Ainsi, ce n'est pas tant le fait d'avoir de la terre qui permet d'accroître de façon notable ses surfaces cacaoyères. C'est surtout la capacité de payer de la main-d'œuvre qui va faire la différence entre les planteurs dans la concurrence qui se joue dans cette « course à la terre » sur front pionnier. Avec un rendement moyen de $500 \mathrm{~kg} / \mathrm{ha}$ de cacao marchand, un prix de $1000 \mathrm{FCFA} / \mathrm{kg}$ de cacao, et un amortissement des coûts d'installation de la plantation sur 25 ans, cela permet d'obtenir une valeur ajoutée nette annuelle de 228000 FCFA/ha. Trois années de production sont donc actuellement suffisantes pour rembourser l'investissement consacré à la plantation. Les investisseurs qui disposent de capitaux financiers trouvent ainsi dans la production cacaoyère une activité qui procure un retour sur investissement lucratif et leur permettant de diversifier leur portefeuille d'activités.

\begin{tabular}{|c|c|c|c|}
\hline \multicolumn{4}{|c|}{$\begin{array}{l}\text { Tableau IV. } \\
\text { Importance relative des différentes formes de production agricole pour les zones d'Obala } \\
\text { et de Talba en pourcentage de la surface cacaoyère totale de la zone. }\end{array}$} \\
\hline & & Formes de productio & \\
\hline \multicolumn{4}{|l|}{ Zone d'Obala } \\
\hline$\%$ du nombre de planteurs & 82 & 17 & 1 \\
\hline \% des surfaces cacaoyères & 71 & 25 & 4 \\
\hline \multicolumn{4}{|l|}{ Zone de Talba } \\
\hline$\%$ du nombre de planteurs & 64 & 28 & 8 \\
\hline \% des surfaces cacaoyères & 30 & 42 & 28 \\
\hline
\end{tabular}

Tableau V.

Impact des revenus non agricoles sur les capacités d'accumulation en cacaoyères.

Surfaces moyennes possédées en cacao (ha)

Exploitants sans revenus non agricoles (« planteur pur »)

Exploitants avec revenus non agricoles

Faibles (< 500000 FCFA/an)

Moyens (500 000 à 2,5 millions FCFA/an)

Élevés (> 2,5 millions FCFA/an)

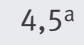

$4,7^{a}$

$14,5^{\mathrm{b}}$

$88,2^{c}$

a, b, $c=$ groupes statistiquement distincts pour le test de Fischer pour un intervalle de confiance de $95 \%$. 


\section{Discussion}

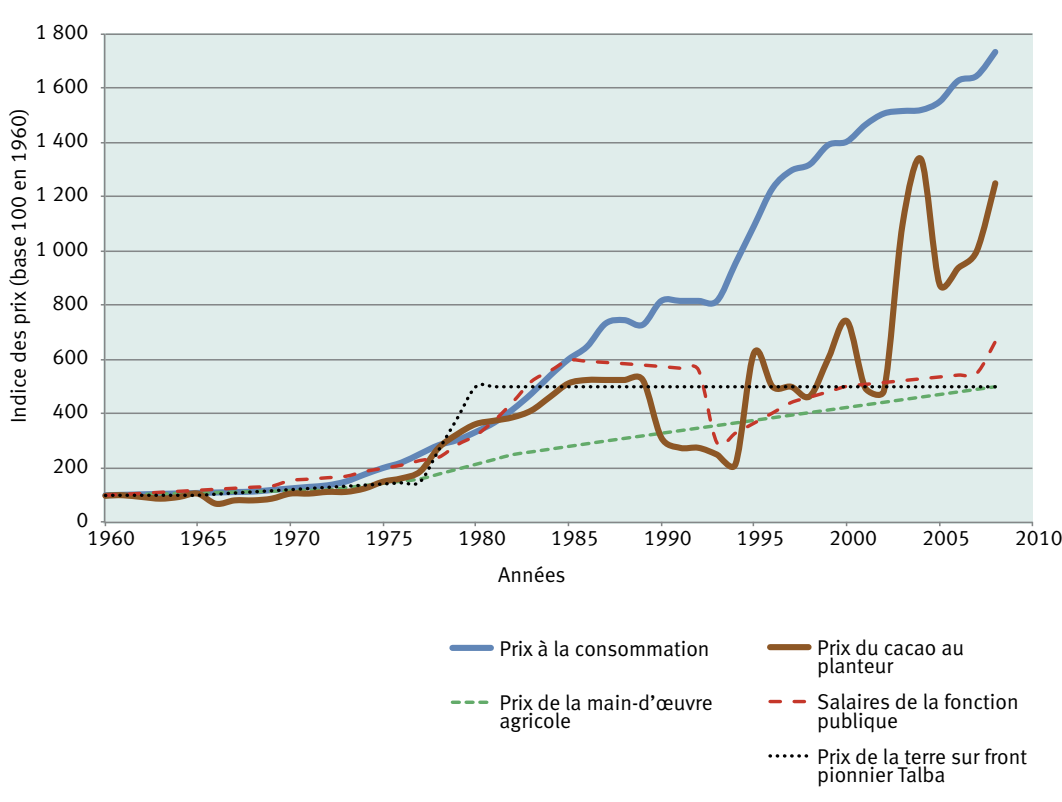

Figure 3.

Comparaison de l'évolution entre 1960 et 2009

des différents indices de prix.

Sources:

Prix à la consommation : Banque mondiale, 2010 ;

Prix de la main-d'œuvre agricole : enquêtes de la présente étude et Losch et al. (1991) ;

Prix du cacao au planteur : enquêtes de la présente étude et Assoumou (1977), Varlet (1997), Kamdem (2010) ;

Salaires de la fonction publique : (DSCN, 1983 ; DSCN, 1991 ; INS, 2000) et fiches du Ministère de la Fonction publique pour les années 2000 à 2009 ;

Prix de la terre sur front pionnier Talba : enquêtes de la présente étude.

En comparant l'évolution entre 1960 et 2010 de différents indices de prix, il apparaît qu'à partir des années 1980 , les prix à la consommation augmentent bien plus rapidement que le prix de la main-d'œuvre agricole ou que les salaires des fonctionnaires et le prix de la terre (figure 3 ). Les hommes d'affaires, dont les activités urbaines et les revenus suivent l'évolution du coût de la vie, vont ainsi pouvoir bénéficier de l'écart grandissant entre le niveau de leurs revenus monétaires et le coût de la terre et de la force de travail agricole. La remontée des prix du cacao à partir de 2003 va encore conforter leur capacité et leur intérêt à investir dans cette filière. Le maintien de niveaux de rémunération faibles pour les revenus du travail agricole ou urbain avantage les détenteurs de capitaux financiers. Il contraint les actifs ruraux ou urbains à accepter une baisse progressive de leur pouvoir d'achat. Les émeutes dites « de la faim » de 2008 ont été suivies par un relèvement du salaire des fonctionnaires ; mais celui-ci n'a pas réellement remis en cause cette tendance générale. Cela laisse supposer, compte tenu du nombre important de jeunes peu qualifiés se retrouvant chaque année sur le marché de l'emploi camerounais, que cette tendance est appelée à se maintenir.

\section{Une augmentation de la production cacaoyère par accroissement des surfaces}

Les rendements en cacao marchand obtenus par les planteurs enquêtés, soit $258 \mathrm{~kg} / \mathrm{ha}$ pour l'ancienne zone d'Obala, et $512 \mathrm{~kg} /$ ha pour le front pionnier de Talba, ne traduisent pas une amélioration notable des rendements. Losch et ses collaborateurs indiquaient déjà en 1992 que la vieille zone cacaoyère du Centre-Sud du Cameroun présentait des rendements qui allaient selon les sources de 200 à $400 \mathrm{~kg}$ et qu’ils étaient pour le département de La Lékié, auquel appartient Obala, de l'ordre de 300 à 350 $\mathrm{kg} / \mathrm{ha}$. Selon cette même source, les rendements plus élevés et de l'ordre de 400 à 600 kg/ha caractérisaient les zones de front pionnier du bassin du Sud-Ouest bénéficiant de cacaoyers plus jeunes et de terres fertiles (Losch et al., 1992).

Les importantes dynamiques de plantation observées sur Talba montrent que, pour les deux zones d'enquête, l'accroissement productif repose avant tout sur une extension de la surface du verger cacaoyer.

Le processus d'augmentation de la production cacaoyère camerounaise par accroissement des surfaces caractérise la période 1910-1963 (Assoumou, 1977). II reste présent même durant la période 1963-1993 où est observable une stagnation de la production camerounaise, et où l'expansion des surfaces plantées dans le bassin cacaoyer du Sud-Ouest du Cameroun compense la baisse de production que connaît durant cette période le vieux bassin cacaoyer du Centre Sud (Varlet, 2000). Le poids relatif de la production cacaoyère issue du Sud-Ouest passera ainsi entre 1963 et 1991 de 12,5\% à $42 \%$ de la production nationale. Ces dynamiques de plantation connaissent un nouvel élan durant la période 1993-2013 dans les deux principaux bassins cacaoyers camerounais, comme en attestent les études portant sur celui du Sud-Ouest et en particulier sur le département de la Mémé (Folefack, 2010 ; Mathey et Pascaud, 2010), et sur celui du Centre-Sud (Dounias, 2000 ; Elong, 2004 ; Manga Essouma, 2013). Si la répartition exacte de la production cacaoyère camerounaise entre bassins de production, entre départements et entre arrondissements est, depuis 1991, rendue problématique par l'absence de recensement agricole et d'enregistrement des origines géographiques des tonnages de cacao collectés, les différents documents consultés s'accordent sur l'importance des dynamiques de plantation cacaoyère et confortent l'idée d'un accroissement de la production camerounaise reposant majoritairement sur une augmentation des 
surfaces plantées.

Donc, dans ces zones d'étude se retrouvent des processus de développement de cette filière reposant sur la mise en valeur de nouvelles terres forestières initialement peu habitées, et sur la migration de la force de travail vers ces fronts pionniers. De tels processus ont déjà été mis en évidence au Cameroun et dans d'autres grands pays producteurs de cacao comme la République de Côte d'Ivoire ou le Ghana (Ruf, 1995). Par contre, ce qui est plus spécifique de la situation étudiée et moins décrit dans la littérature, c'est le fait que le développement de ces nouvelles surfaces cacaoyères s'accompagne depuis le début des années 1980 d'une montée en puissance des formes patronale et capitaliste d'une production cacaoyère historiquement portée par une agriculture de type familial (Léna, 1979).

\section{Une transition capitaliste peu attendue}

L'histoire du développement de la production cacaoyère au Cameroun, comme celle des autres pays producteurs d'Afrique de l'Ouest, a reposé davantage sur l'essor d'une petite agriculture familiale marchande (Lesourd, 1982) que sur des processus d'accumulation en capitaux financiers ou l'émergence de formes patronale ou capitaliste de la production agricole. Weber, travaillant sur le bassin cacaoyer du Centre-Cameroun, concluait en $1974:$ : ... les rapports de production au sein du milieu rural et la forme d'accumulation à laquelle ils aboutissent montrent bien que cette insertion dans les rapports internationaux de production se satisfait de la non émergence de rapports capitalistes de production, et de l'inexistence d'une accumulation productive. " (Weber, 1974). Leplaideur s'interrogeait en 1989 , non sur les possibilités d'accumulation des planteurs de cacao du CentreCameroun, mais sur leur simple capacité de reproduction mise à mal par l'augmentation des densités de population et la baisse de leur pouvoir d'achat (Leplaideur, 1989). Ces observations sont confirmées par les données des deux recensements agricoles disponibles pour le Cameroun. Elles montrent une diminution du capital « surfaces cacaoyères " qui passe pour les planteurs camerounais de 1,6 ha/exploitation en 1972 à 1,3 ha/exploitation en 1984 (DNRA, 1987; PNUD-FAO, 1977). Les différentes études réalisées durant les années de crise et d'« ajustement structurel ${ }^{1}$ " (1987 à 1995) indiquent qu'au Cameroun, comme dans de nombreux pays d'Afrique tropicale, la plupart des exploitants agricoles ont développé des stratégies « défensives » et des pratiques techniques « extensives » (Alary, 1996 ; Courade, 1994 ; Losch et al., 1991) qui n'ont guère permis une accumulation en capital cacaoyer.

Les résultats de la présente étude montrent que ces observations restent vraies en 2012 pour la zone cacaoyère historique d'Obala, où les petites ( 0,1 à 2 ha) et moyennes (2 à 6 ha) exploitations familiales restent numériquement majoritaires (97\%) et concentrent l'essentiel des surfaces

${ }^{1}$ Un programme d'ajustement structurel est un programme de réformes économiques que le Fonds monétaire international (FMI) ou la Banque mondiale (BM) mettent en place pour aider les pays touchés par de grandes difficultés économiques à sortir de la crise qu'ils traversent. Ces programmes s'appuient sur la réduction des dépenses publiques, la libéralisation de l'économie nationale et une ouverture sur le marché international.
BOIS ET FORÊTS DES tROPIQUES, $2014, N^{\circ} 321$ (3)
CACAOYERS AU CENTRE-CAMEROUN / LE POINT SUR...

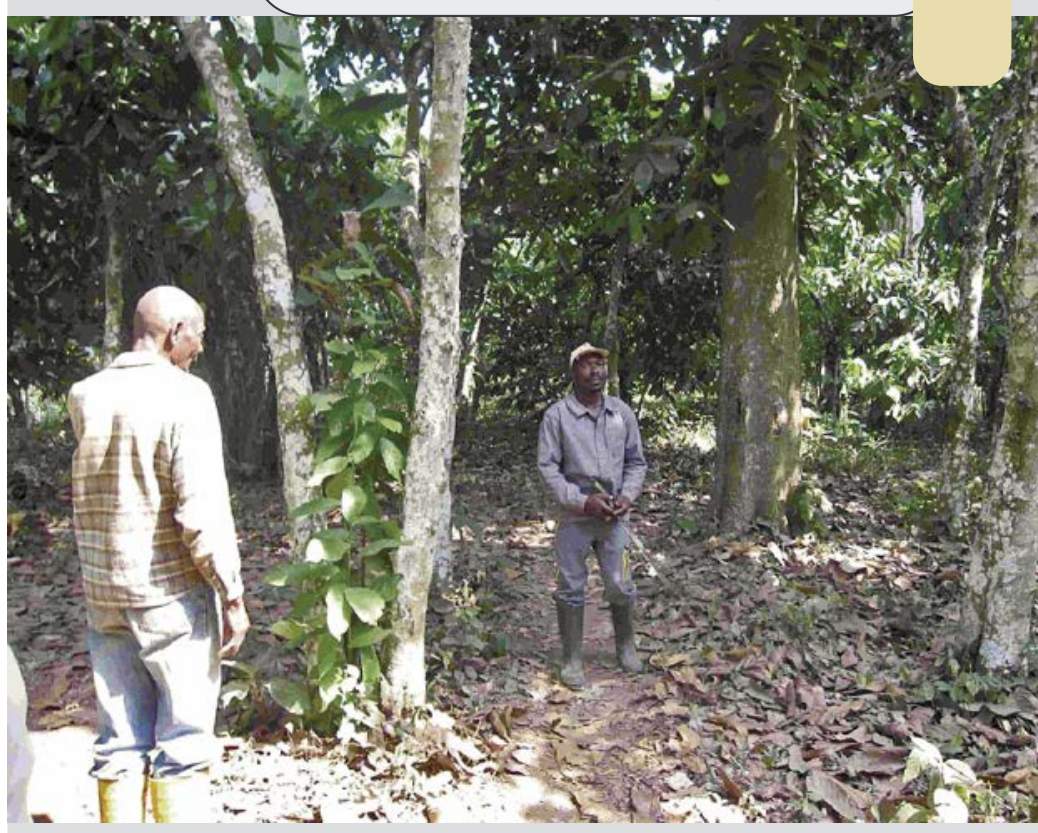

Photo 2.

Agroforêt à base de cacao de la zone d'Obala.

Photo P. Pédelahore.

cacaoyères de l'arrondissement (86\%).

Par contre, les résultats montrent que ces observations doivent être révisées et actualisées pour ce qui concerne la zone de front pionnier de Talba. Dans cette zone, la présente étude révèle l'émergence d'exploitations cacaoyères de grande taille (6 à $30 \mathrm{ha}$ ) et de très grande taille ( $30 \mathrm{ha})$. Elles représentent $30 \%$ du nombre total des exploitations, contrôlent $75 \%$ des surfaces cacaoyères et portent la surface moyenne par exploitation à 8,21 ha. Ils confirment les rares indications disponibles sur les structures des exploitations de cette zone qui chiffraient à 36,5\% l'importance numérique des exploitations d'une surface cacaoyère supérieure à 5 ha (Jagoret et al., 2006) et à 8 ha la surface cacaoyère moyenne par exploitation (Elong, 2004).

Ces résultats montrent la coexistence dans la zone de Talba de formes familiale, patronale et capitaliste de la production agricole. En effet, depuis le début des années 1980, il est observé un important développement du marché foncier et du salariat agricole. Ces évolutions sont accompagnées d'une montée en puissance des formes patronale et capitaliste de la production qui contrôlent aujourd'hui 70 \% du capital cacaoyer planté. Cette transformation des formes et des rapports de production par l'introduction dans la société agraire de capitaux financiers correspond à ce que Castex (1977) définit comme un processus de transition capitaliste.

Pour Castex (1977), les « planteurs moyens » de notre typologie constituent l'archétype de "l'agriculture familiale ». Selon cet auteur, les autres types d'exploitants agricoles et les ouvriers agricoles découlent de ce type central par perte, ou accumulation progressive (capitalisation), des moyens de production. Cette évolution aboutit ainsi à la constitution de la classe des prolétaires, peu à peu privés de leurs moyens de production et contraints à vendre leur force de travail, et à celle des capitalistes qui ont progressivement accaparé la majeure partie des moyens de production. 


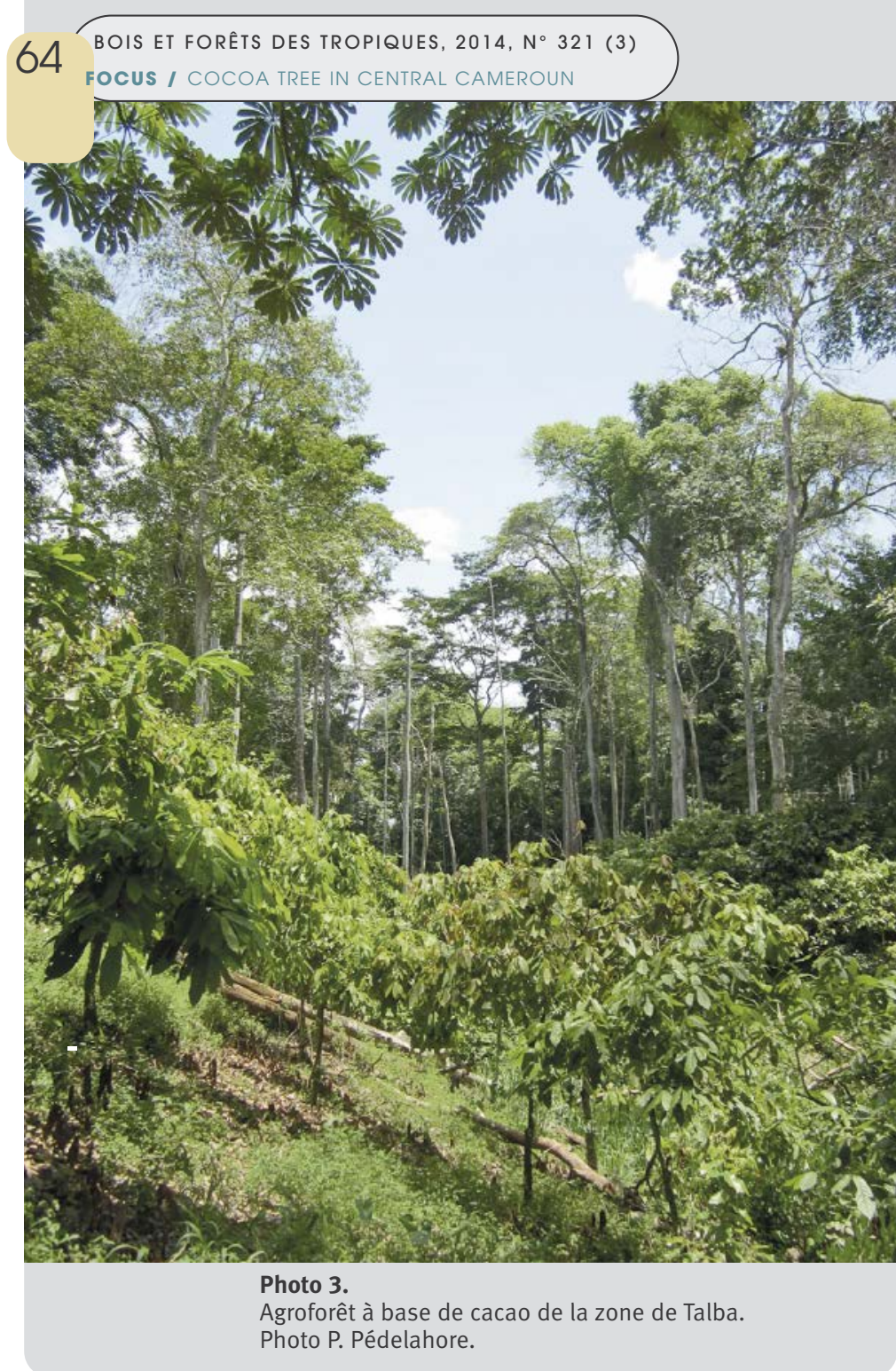

Cependant, dans la situation de la zone de Talba, la majorité des ouvriers agricoles viennent de la province du Nord-Ouest et n'ont pas été directement spoliés de leurs terres par les grands planteurs aujourd'hui installés dans la zone de Talba. Ces ouvriers agricoles sont venus chercher dans la zone de Talba des revenus monétaires plus importants que ceux auxquels ils pouvaient prétendre dans leur province d'origine. Certains d'entre eux parviendront d'ailleurs à se constituer une certaine épargne et à devenir « planteurs » à leur tour, même si leur nombre reste marginal. De même, les petits planteurs de la zone d'Obala ne sont pas contraints de venir louer leurs bras dans les grandes plantations cacaoyères de la zone de Talba. Nombre d'entre eux développent des activités non agricoles, souvent en milieu urbain (Pédelahore et al., 2011), leur permettant de subvenir à leur besoins essentiels et de réussir parfois à développer leurs propres trajectoires d'accumulation. Enfin, la majorité des capitaux des grands et très grands planteurs proviennent d'activités non agricoles antérieures à l'installation de leurs plantations et à l'exploitation de la main-d'œuvre salariée agricole. Ainsi, même si l'on peut considérer que la zone de Talba connaît bien des rapports de production de type prolétaires/capitalistes, nous ne sommes pas ici dans un système fermé sur l'extérieur, rigide et inéluctable de type latifundium-minifundium tel que le décrit par Castex (1977) pour le Chili.

C'est que cette transition capitaliste s'effectue au Cameroun dans un monde socio-économique où la mobilité professionnelle et spatiale des individus et celle des capitaux sont bien plus importantes que celles décrites pour l'expérience chilienne. Ces réalités ne sont pas propres à la situation camerounaise. Le développement des revenus non agricoles des exploitants agroforestiers familiaux (Soini, 2005) et l'investissement des capitaux financiers dans l'agriculture (AREION group, 2012) sont aujourd'hui largement répandus.

De même, la marchandisation du travail et de la terre n'est pour l'instant pas totale. Le capital social et institutionnel continue de jouer un rôle important dans ces sociétés et ces territoires agraires, en particulier pour l'accès à la terre. L'avenir de cette évolution des formes de production dira si les singularités de cette transition capitaliste sont liées au fait que ce processus n'est pas achevé, ou si elles sont le signe du développement d'un capitalisme endogène africain spécifique.

\section{Conclusion}

Au Cameroun, comme dans de nombreux pays d'Afrique, le développement de la cacaoculture a longtemps été basé sur des exploitations de type familial, l'extension des surfaces plantées et la migration des planteurs vers les fronts pionniers forestiers. Depuis le début des années 1980 , les dynamiques que connaissent les systèmes agroforestiers à base de cacao de la zone de Talba montrent la montée en puissance des formes patronale et capitaliste de la production agricole. Elles s'appuient sur des capitaux financiers majoritairement d'origine urbaine et relevant de secteurs d'activités non agricoles. Les résultats de l'étude montrent que, si la mobilité de la force de travail reste toujours une composante essentielle des dynamiques des systèmes agroforestiers (SAF) à cacaoyers, la mobilité des capitaux financiers d'origine urbaine devient également l'un des déterminants important de ces dynamiques.

Ces résultats incitent à analyser l'évolution de ces systèmes agroforestiers en portant une plus grande attention qu'avant à l'importance relative et aux interactions entre les différentes formes de production (familiale, patronale, capitaliste) et entre les trajectoires d'accumulation en capital qui se développent en milieu rural et en milieu urbain. Cela milite également pour conduire des études dans d'autres zones du Cameroun pour mesurer l'évolution éventuelle de l'importance relative des différentes formes de production (familiale, patronale, capitaliste). Cela permettra de voir si les changements identifiés sur Talba sont inféodés à cette zone ou s'ils annoncent une évolution plus générale des SAF à cacaoyers au Cameroun et dans d'autres pays d'Afrique. 


\section{Références bibliographiques}

ALARY V., 1996. Incertitude et prise de risque en période d'ajustement. Le comportement des producteurs de cacao du Cameroun avant et après 1994. Thèse doctorat, Université Paris I-Panthéon Sorbonne, France, 750 p.

AREION GROUP, 2012. Le land grabbing : la course aux terres fertiles. Carto : le monde en cartes, $9:$ 54-57.

ASSOUMOU J., 1977. L'économie du cacao. Agriculture d'exportation et bataille du développement en Afrique tropicale. Paris, France, Jean-Pierre Delarge.

BANQUE MONDIALE, 2010. Séries statistiques du Cameroun. CD de données statistiques disponible aux Bureaux de la Banque mondiale, Quartier Bastos, Yaoundé, Cameroun.

BERGERET P., DUFUMIER M., 2002. Analyser la diversité des exploitations agricoles. In : Mémento de l'ingénieur agronome. Paris, France, Cirad/Gret/Ministère des Affaires étrangères, 411-432.

CASTEX P., 1977. Voie chilienne au socialisme et luttes paysannes. Approche théorique et pratique d'une transition capitaliste non révolutionnaire. Paris, France, François Maspero.

COURADE G., 1994. Le village camerounais à l'heure de l'ajustement. Paris, France, Karthala.

CTA, 2012. Secteur du cacao. Agritrade. Le commerce ACP analysé et décrypté.

DE SOUZA H. N., DE GRAAFF J., PULLEMAN M. M., 2012. Strategies and economics of farming systems with coffee in the Atlantic Rainforest Biome. Agroforestry Systems, 84 (2): 227-242.

DNRA, 1987. Recensement agricole 1984. Secteur traditionnel. Volume 1. Résultats du niveau national. Yaoundé, Cameroun, Direction nationale du recensement agricole, ministère de l'Agriculture, $1: 255 \mathrm{p}$.

DOUNIAS E., 2000. Écotone forêt-savane et système agraire des Tikar du Haut Mbam (Cameroun central). In : Servant M., Servant-Vildary S. (éds). Dynamiques à long terme des écosystèmes forestiers intertropicaux. Paris, France, IRD/Unesco, 85-102.

DSCN, 1983. Annuaire statistique du Cameroun. Yaoundé, Cameroun, Direction de la statistique et de la comptabilité nationale, ministère du Plan et de l'Aménagement du Territoire.

DSCN, 1991. Annuaire statistique du Cameroun. Yaoundé, Cameroun, Direction de la statistique et de la comptabilité nationale, ministère du Plan et de l'Aménagement du Territoire.

ELONG J. G., 2004. Éton et Manguissa, de la Lékié au Mbamet-Kim : jeux et enjeux fonciers (Centre-Cameroun). Afriques, Les Cahiers d'Outre-Mer, 226-227 : 1-15.

ELOY L., 2008. Dynamiques et adaptation des systèmes agroforestiers périurbains dans le Nord-Ouest amazonien. Bois et Forêts des Tropiques, 296 : 39-49.
FOLEFACK D. P., 2010. Pauvreté et répartition des revenus en zone cacaoyère du Cameroun. Tropicultura, 28 (1) : 5-9.

GAFSI M., 2006. Exploitation agricole et agriculture durable. Cahiers Agricultures, 15 (6) : 491-497.

GARRITY D. P., 2004. Agroforestry and the achievement of the Millenium Development Goals. Agroforestry Systems, 61-62 (1-3): 5-17.

GHIGLIONE R., MATALON B., 1998. Les enquêtes sociologiques. Théories et pratiques. Paris, France, Armand Colin.

GRIFFON M., 2002. Révolution Verte, Révolution Doublement Verte. Quelles technologies, institutions et recherche pour les agricultures de l'avenir ? Mondes en développement, $117: 39-44$.

INS, 2000. Annuaire statistique du Cameroun. République du Cameroun, Institut national de la statistique (INS).

JAGORET P., 2011. Analyse et évaluation de systèmes agroforestiers complexes sur le long terme : application aux systèmes de culture à base de cacaoyer au Centre-Cameroun. Thèse de doctorat, Montpellier SupAgro, France, 235 p.

JAGORET P., COUVE C., BOUAMBI E., MENIMO T., DOMKAM I., NYASSÉ S., 2006. Caractérisation des systèmes de cacaoculture du Centre-Cameroun. Yaoundé, Cameroun, CiradIrad-MAEE, $101 \mathrm{p}$.

KAMDEM C., 2010. L'économie du cacao au Cameroun. Université de Yaoundé II SOA, Cameroun.

LANDAIS E., 1996. Typlologies d'exploitations agricoles. Nouvelles questions, nouvelles méthodes. Économie Rurale, $236: 3-15$.

LÉNA P., 1979. Transformation de l'espace rural dans le front pionnier du Sud-Ouest ivoirien. Thèse de doctorat $3^{\mathrm{e}}$ cycle, Université Paris X-Nanterre, France, 345 p.

LEPLAIDEUR A., 1989. Vie et survie domestique en zone forestière camerounaise : la reproduction simple est-elle assurée ? In : Eldin M., Milleville P. (éds). Le risque en agriculture. Paris, France, Orstom, coll. À travers champs, 227 290.

LESOURD M., 1982. L'émigration Baoulé vers le Sud-Ouest de la Côte d'Ivoire. Thèse de doctorat $3^{\mathrm{e}}$ cycle, Université Paris X-Nanterre, France, $526 \mathrm{p}$.

LOSCH B., DAVIRON B., FREUD C., GERGELY N., 1992. Relance régionalisée de la production paysanne de café et de cacao au Cameroun. Étude de faisabilité. Phase 1 : Cadrage général de la relance. Yaoundé, Cameroun, Cirad/Sofreco, 87 p.

LOSCH B., FUSILLIER J.-L., DUPRAZ P., 1991. Stratégies des producteurs en zone caféière et cacaoyère du Cameroun. Quelles adaptations à la crise ? Montpellier, France, CiradDSA, coll. Documents Systèmes agraires, 12, 252 p.

MANGA ESSOUMA F., 2013. Systèmes agroforestiers à base de cacaoyers : Dynamiques et stratégies des acteurs à Akongo (région du Centre-Cameroun). Université de Yaoundé 1, Cameroun, $107 \mathrm{p}$. 
MATHEY E., PASCAUD C., 2010. Comment améliorer la compétitivité des exploitations agricoles face à l'avancée du front pionnier? Mise en oeuvre d'un diagnostic agraire dans l'arrondissement de Konye (Cameroun). Montpellier, France, SupAgro/IRC, 147 p.

NGONO F., 2013. Systèmes agroforestiers cacao : évolution et stratégie des acteurs dans le village de Talba. Mémoire de stage d'ingénieur, Université de Yaoundé 1/Cirad/IRD, Cameroun, $77 \mathrm{p}$.

OLIVIER DE SARDAN J.-P., 1995. La politique du terrain. Sur la production des données en anthropologie. Enquête, Les terrains de l'enquête, $1: 71-109$.

ORSTOM, 1959. Atlas du Cameroun. Planche phytogéographie au 1/2 000 000. Bondy, France, Orstom.

PÉDELAHORE P., 2014. Farmers accumulation strategies and agroforestry systems intensification: The example of cocoa in the Central region of Cameroon over the 1910-2010 period. Agroforestry Systems, 88 (6): 1157-1166..

PÉDELAHORE P., 2012. Stratégies d'accumulation des exploitants agricoles : l'exemple des cacaoculteurs du CentreCameroun de 1910 à 2010. Thèse de doctorat en géographie, Université de Toulouse-Le Mirail, France, 440 p.

PÉDELAHORE P., TCHATCHOUA R., TONKA M., NTSAMA M., ANDRIEU N., 2011. Resituer l'adoption des propositions techniques de la recherche dans les stratégies d'adaptation des exploitants agricoles familiaux. Revue d’Élevage et de Médecine Vétérinaire des Pays Tropicaux, 64 (1-4) : 33-41. [En ligne] http://remvt. cirad.fr/CD/derniers num/2011/REMVT11 14.pdf.

PERROT C., 1990. Typlogie d'exploitations construite par agrégation autour de pôles définis à dire d'experts. Inra Productions Animales, 3 (1) : 51-66.

PNUD-FAO, 1977. Recensement mondial de l'agriculture et établissement d'un système permanent de statistiques courantes. Cameroun, Congo, Gabon, Empire Centrafricain, Tchad. Résultats du recensement agricole 1972/1973 pour le Cameroun. Pnud/FAO, $360 \mathrm{p}$.

PNUD, 2000. Études socio-économiques régionales au Cameroun. Éradication de la pauvreté. Amélioration des données sociales. Province du Centre. République du Cameroun, Ministère des Investissements publics et de l'Aménagement du Territoire, Programme des Nations unies pour le développement, Bureau des services d'appui aux projets, $156 \mathrm{p}$.

RUF F., 1995. Booms et crises du cacao. Les vertiges de l'or brun. Paris, France, Karthala/Cirad/Ministère de la Coopération.

SABOURIN E., CARON P., TONNEAU J.-P., 2004. Dynamiques territoriales et trajectoires de développement local : retour d'expériences dans le Nordeste brésilien. Cahiers Agricultures, 13 (6) : 539-545.

SANTOIR C., BOPDA A., 1995. Atlas régional. Sud-Cameroun. Paris, France, Orstom.

SOINI E., 2005. Changing livelihoods on the slopes of Mt. Kilimanjaro, Tanzania: Challenges and opportunities in the Chaga homegarden system. Agroforestry Systems, 64 (2): 157-167.
SONWA D. J., WEISE S. F., NDOYE O., JANSSENS M., 2003. Promotion des agroforêts cacao en Afrique de l'Ouest et Centrale (AOC). XII Congrès forestier mondial, Québec City, Canada, 21-28 septembre 2003.

VARLET F., 1997. Réformes des institutions dans les filières cacao et café au Cameroun. Chronique des années 1990 à 1997. Montpellier, Cirad-Sar, 55 p.

VARLET F., 2000. Institutions publiques et croissance agricole au Cameroun. Thèse agro-économie. Ensa de Montpellier, France, $467+320 \mathrm{p}$.

WEBER J., 1974. Types de surproduit et formes d'accumulation. La province cacaoyère du centre-sud Cameroun. Yaoundé, Cameroun, Orstom, 69-85. 